\title{
The FRED for Cerebral Aneurysms of the Posterior Circulation: A Subgroup Analysis of the EuFRED Registry
}

\author{
(D) C.J. Griessenauer, (D) M.A. Möhlenbruch, (DP. Hendrix, (D). Ulfert, (D). Islak, (D) M. Sonnberger, (D). Engelhorn, \\ (D) E. Müller-Thies-Broussalis, (D)T. Finkenzeller, (D) M. Holtmannspötter, (D).-H. Buhk, (DW. Reith, (D) A. Simgen, (D) H. Janssen, \\ (D) N. Kocer, and (D) Killer-Oberpfalzer
}

\begin{abstract}
BACKGROUND AND PURPOSE: Flow diversion for the posterior circulation remains a promising treatment option for selected posterior circulation aneurysms. The Flow-Redirection Intraluminal Device (FRED) system has not been previously assessed in a large cohort of patients with posterior circulation aneurysms. The purpose of the present study was to assess safety and efficacy of FRED in this location.
\end{abstract}

MATERIALS AND METHODS: Consecutive patients with posterior circulation aneurysms treated at 8 centers participating in the European FRED study (EuFRED) between April 2012 and January 2019 were retrospectively reviewed. Complication and radiographic and functional outcomes were evaluated.

RESULTS: Eighty-four patients (median age, 54 years) with 84 posterior circulation aneurysms were treated with the FRED. A total of 25 aneurysms (29.8\%) had previously ruptured, even though most aneurysms were diagnosed incidentally (45.2\%). The intradural vertebral artery was the most common location (50\%), and saccular, the most common morphology (40.5\%). The median size was $7 \mathrm{~mm}$. There were 8 (9.5\%) symptomatic thromboembolic and no hemorrhagic complications. Thromboembolic complications occurred mostly $(90.9 \%)$ in nonsaccular aneurysms. On last follow-up at a median of 24 months, $78.2 \%$ of aneurysms were completely occluded. Functional outcome at a median of 27 months was favorable in $94 \%$ of patients. All mortalities occurred in patients with acute subarachnoid hemorrhage and its sequelae.

CONCLUSIONS: The largest cohort of posterior circulation aneurysms treated with the FRED to date demonstrated favorable safety and efficacy profiles of the device for this indication. Treatment in the setting of acute subarachnoid hemorrhage was strongly related to mortality, regardless of whether procedural complications occurred.

$F^{2}$ ow diversion for aneurysms of the posterior circulation continues to be more controversial than other endovascular treatment modalities in that location. Whereas it is established in the anterior circulation, flow diversion in the posterior circulation remains "off-label" according to the FDA, which has not granted approval to any flow-diverting device for that indication. Still, flow-diverting stents are used at increasing frequency in those locations. ${ }^{1-3}$ The

Received October 23, 2019; accepted after revision January 14, 2020.

From the Research Institute of Neurointervention (C.J.G., E.M.-T.-B., M.K.-O.), Paracelsus Medical University, Salzburg, Austria; Department of Neurosurgery (C.J.G.), Geisinger, Danville, Pennsylvania; Department of Neuroradiology (M.A.M., C.U.), Heidelberg University Hospital, Heidelberg, Germany; Department of Neurosurgery (P.H.), Saarland University Medical Center and Saarland University Faculty of Medicine, Homburg/Saar, Germany; Department of Neuroradiology (C.I., N.K.), Cerrahpasa Medical School, Istanbul University, Istanbul, Turkey; Department of Neuroradiology (M.S.), Kepler Universitätsklinikum, Linz, Austria; Department of Neuroradiology (T.E.), University Hospital Erlangen, Erlangen, Germany; Institute of Radiology and Neuroradiology (T.F., M.H.), Klinikum

Nuernberg Sued, Paracelsus Medical University, Nuernberg, Germany; Department of Neuroradiology (T.F.), Klinikum Weiden, Weiden, Bavaria, Germany; Department of Neuroradiology (J.-H.B.), University Hospital Hamburg Eppendorf, Hamburg, Germany; Clinic for Diagnostic and Interventional Neuroradiology (W.R., A.S.), Universitätsklinikum des Saarlandes, Homburg/Saar, Homburg, Saarland, Germany and Institute for Neuroradiology (H.J.), Klinikum Ingolstadt, Ingolstadt, Germany.

Due to equal contributions, the 2 last authors share senior authorship. largest studies on posterior circulation flow diversion are currently available for the Pipeline Embolization Device (PED; Covidien, Irvine, California). ${ }^{4,5}$ Aneurysms of the vertebral artery have been found to be particularly amenable to flow diversion. ${ }^{5,6}$ Larger cohorts of other flow diverters used in the posterior circulation are currently lacking. Here, we performed a subgroup analysis of posterior circulation cases treated with the Flow-Redirection Endoluminal Device (FRED; MicroVention, Tustin, California) at centers participating in the European FRED study (EuFRED). ${ }^{7}$

\section{MATERIALS AND METHODS Study Design and Data Collection}

The EuFRED study was a retrospective, multicenter postmarket registry of consecutive patients with intracranial aneurysms treated with the FRED at European high-volume neurovascular

Please address correspondence to Christoph J. Griessenauer, MD, Department of Neurosurgery, Geisinger, 100 N Academy Ave, Danville, PA 17822; e-mail: christoph.griessenauer@gmail.com; @cgriessenauer

\section{Indicates article with supplemental on-line table.}

http://dx.doi.org/10.3174/ajnr.A6447 

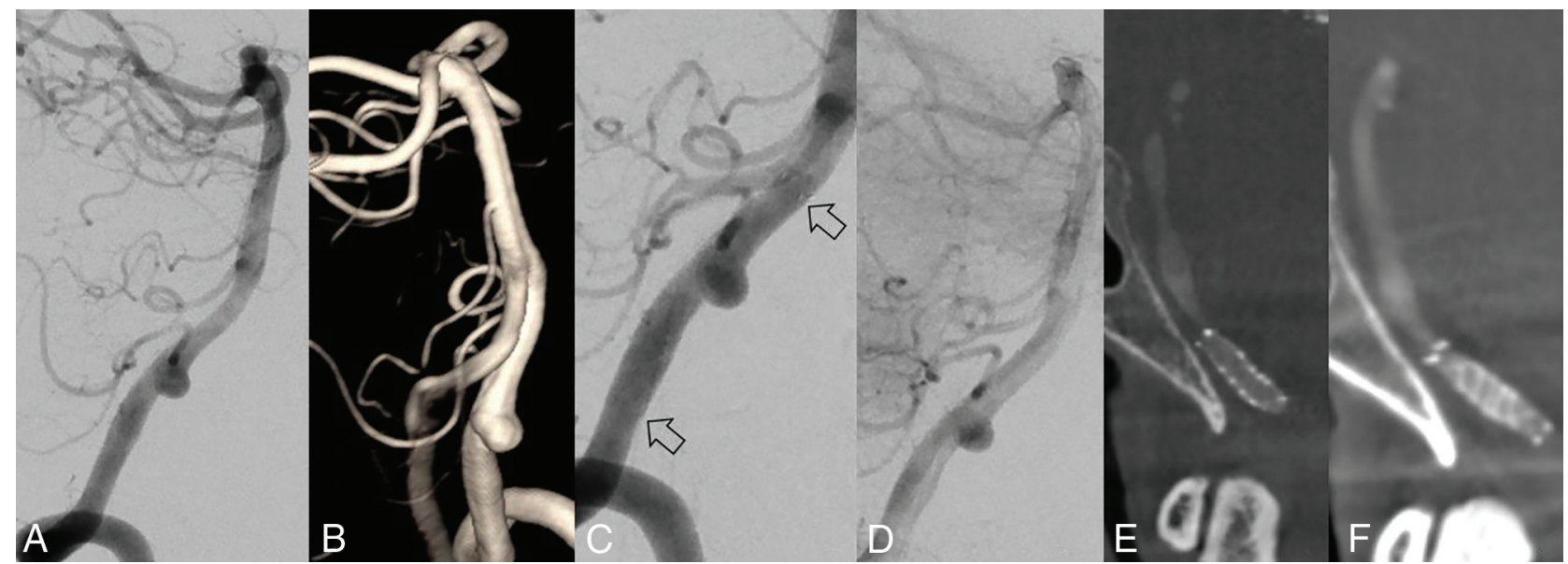

FIG 1. A 66-year-old woman presenting with an unruptured left vertebral artery aneurysm measuring 5 mm in maximum diameter. A, Lateral view of a left vertebral artery digital subtraction angiogram. B, Rotational 3D reconstruction. $C$, Angiogram post-FRED placement (arrows show the proximal and distal ends of the flow diverter). $D$, Stasis in the aneurysm after flow-diverter placement. $E$, Conebeam CT with contrast 1 month after stent placement shows complete occlusion of the aneurysm. $F$, Confirmation of aneurysm occlusion at 1 year.

centers as previously described. ${ }^{7}$ The decision to use the FRED was at practitioner discretion to reflect real-world indications. An independent study nurse not involved in the clinical decisionmaking collected all raw data of the original EuFRED dataset. For this subgroup analysis of the posterior circulation aneurysms treated, 8 centers that had treated posterior circulation aneurysms provided additional consecutive cases up to January 2019. Aneurysms were classified according to the morphology as saccular, fusiform, dissecting, and blister/pseudoaneurysm with and without intraluminal thrombus, with the maximum diameter measured in millimiters. The categorization of aneurysm morphology was performed at the individual centers and in accordance with prior classification of posterior circulation aneurysms treated with flow diversion. ${ }^{5}$ Details on platelet function testing and antiplatelet regimen were obtained. Complications were classified as thromboembolic, hemorrhagic, and other and divided in symptomatic and asymptomatic. Clinical and imaging follow-up time points were at the discretion of the participating center as was the imaging technique. Angiographic outcome was evaluated locally at the individual center by a radiologist not involved in the procedure and graded as completely occluded, near-completely occluded with neck remnant, or incompletely occluded. Functional outcomes were assessed locally and by using the mRS. Internal review board approval was obtained at all participating institutions, and all patients signed approved consent forms.

\section{Statistical Analyses}

Continuous variables are presented as median and range, and categoric variables are presented as frequency and percentage. Analyses were performed using $\chi^{2}$ and Fisher exact tests. $P$ values $<.05$ were considered statistically significant.

\section{RESULTS}

\section{Baseline Patient and Aneurysm Characteristics}

A total of 84 aneurysms in 84 patients (median age, 54 years) were treated with the FRED. All patients with electively treated aneurysms had a good preprocedural functional status (mRS, $0-2)$. A history of aneurysm rupture was present in 25 (29.8\%) aneurysms, with 12 aneurysms (14.3\%) treated in the acute phase (within 48 hours) of aneurysm rupture. The intradural vertebral artery (50\%) (Fig 1) and basilar artery (26.1\%) were the most common locations of the aneurysm. The aneurysms were saccular (40.5\%), fusiform (25.0\%), dissecting (22.6\%), or blister (11.9\%) and had a median maximum diameter of $7 \mathrm{~mm}$ (range, 2-67 mm). Intraluminal thrombus was present in $8.3 \%$ of aneurysms (Table 1).

\section{Treatment Characteristics and Outcomes}

Most aneurysms were treated with a single FRED (95.2\%). Adjunctive coiling was performed in $16.7 \%$. Platelet function testing was available in $95.2 \%$ of cases and resulted in a regimen change in $2.4 \%$. The most common postprocedural antiplatelet medication regimen was aspirin and clopidogrel (86.9\%). Of the 12 aneurysms treated in the acute setting of subarachnoid hemorrhage, 9 patients (75\%) were started on tirofiban and transitioned to aspirin and clopidogrel (8 patients) or aspirin and ticagrelor (1 patient), while $2(16.7 \%)$ were loaded with aspirin and clopidogrel, and $1(8.3 \%)$, with aspirin and ticagrelor. There were 11 (13.1\%) thromboembolic infarctions, most frequently affecting the cerebellum and occurring mostly in nonsaccular aneurysms (90.9\%). Symptomatic infarctions occurred in 8 (9.5\%) patients. Imaging follow-up was available for 77 (91.7\%) patients. Imaging modalities included MRA (50\%), DSA (45.6\%), conebeam CT with IV contrast (2.9\%), and CTA (1.5\%). At a median follow-up of 24 months, $78.2 \%$ of aneurysms were completely and $9 \%$ nearcompletely occluded. Retreatment with another flow diverter was performed in $7.1 \%$ of patients (Fig 2). Functional outcome at a median of 27 months was favorable in $94 \%$ of patients (mRS, $0-2)$ and available for $98.8 \%$ of patients. There were $5(6 \%)$ mortalities, all occurring in patients treated in the acute setting of subarachnoid hemorrhage. In one of those patients, mortality was due to a thromboembolic infarction from the flow-diversion procedure (Table 2). 
Table 1: Baseline characteristics

\begin{tabular}{lc}
\hline \multicolumn{1}{c}{ Parameter } & No. \\
\hline No. of patients & 84 \\
No. of aneurysms & 84 \\
Females & $42(50.0 \%)$ \\
Median age (range) (yr) & $54(15-81)$ \\
Pretreatment mRS of patients without SAH & 58 \\
0 & $53(91.4 \%)$ \\
1 & $4(6.9 \%)$ \\
2 & $1(1.7 \%)$ \\
$3-5$ & $0(0.0 \%)$ \\
Index aneurysm presentation & \\
Acute SAH (within 48 hours) & $12(14.3 \%)$ \\
<2 weeks since SAH & $6(7.1 \%)$ \\
>2 weeks since SAH & $7(8.3 \%)$ \\
Stroke & $2(2.4 \%)$ \\
Brain stem compression & $5(6.0 \%)$ \\
Brain stem infarction & $2(2.4 \%)$ \\
Incidental & $38(45.2 \%)$ \\
Other & $12(14.3 \%)$ \\
Aneurysm location & \\
Vertebral artery (intradural) & $42(50 \%)$ \\
Basilar artery & $22(26.1 \%)$ \\
Posterior cerebral artery & $7(8.3 \%)$ \\
Superior cerebellar artery & $4(4.8 \%)$ \\
Vertebral artery (extradural) & $4(4.8 \%)$ \\
Posterior inferior cerebellar artery & $3(3.6 \%)$ \\
Vertebrobasilar junction & $2(2.4 \%)$ \\
Aneurysm morphology & \\
Saccular & $34(40.5 \%)$ \\
Fusiform & $21(25.0 \%)$ \\
Dissecting & $19(22.6 \%)$ \\
Blister/pseudoaneurysm & $10(11.9 \%)$ \\
Index aneurysm pretreated & $7(8.3 \%)$ \\
Aneurysm size (median) (range) (mm) & $7(2-67)$ \\
Intra-aneurysmal thrombus & $7(8.3 \%)$ \\
\hline Other & \\
\hline &
\end{tabular}

a Other Headache $(n=7)$, vertigo $(n=1)$, syncope $(n=1)$, TIA (1), dizziness $(n=1)$, Traumatic brain injury $(n=1)$.

${ }^{b}$ Pretreatment endovascular $(n=6)$, endovascular, and open microsurgery $(n=1)$.

\section{Predictors of Complication and Occlusion Status at Last Follow-Up}

The small number of cases with complications and incomplete occlusion status limited the ability to perform statistical analysis. All thromboembolic complications occurred in aneurysms not treated with adjunctive coiling, albeit this relationship did not reach statistical significance. All patients with thromboembolic complications had undergone platelet function testing, and no adjustments to the antiplatelet regimen were warranted. There were no associations of occlusion status or complications with any of the recorded variables (On-line Table).

\section{DISCUSSION}

The present cohort is the largest reported on the FRED for aneurysms of the posterior cerebral circulation. The FRED was successfully implanted in 84 patients with 84 aneurysms, most commonly located along the intradural vertebral artery. Symptomatic thromboembolic complications occurred in $9.5 \%$ of patients, whereas no hemorrhagic complications were reported. Complete or near-complete occlusion was achieved in $87.2 \%$ of aneurysms at a median follow-up of 24 months. At a median clinical follow-up of 27 months, all living patients had a favorable functional outcome. Mortalities occurred only in patients treated in the acute setting of subarachnoid hemorrhage.

\section{The Flow-Redirection Intraluminal Device}

The FRED is a dual-layer braided device with an inner low-porosity stent that acts as the flow diverter and an outer part that serves as a scaffold for the inner stent. The EuFRED study with 579 aneurysms treated with the FRED is the largest to date. In aneurysms followed for $>1$ year, complete occlusion was achieved in $95.3 \%$. Transient morbidity, permanent morbidity, and mortality rates were $3.2 \%, 0.8 \%$, and $1.5 \%$, respectively. ${ }^{7}$

The Italian core lab-adjudicated FRED registry included 165 aneurysms. ${ }^{8}$ Device-related morbidity and mortality occurred in $6.1 \%$ and $2.4 \%$ of patients. Complete or near-complete occlusion at 12 to 24 months was $77 \%$ and $19 \%$, respectively. The study also included 29 aneurysms in the posterior circulation. Even though this subgroup was not discussed in detail, posterior circulation aneurysms contributed to mortality in particular. Among the 6 delayed deaths, 3 occurred in basilar artery aneurysms. ${ }^{8}$

The Safety and efficacy Analysis of FRED Embolic device in aneurysm treatment (SAFE) study is the third largest on FRED, enrolling 103 aneurysms; posterior circulation aneurysms were not included. ${ }^{9}$

In the United States, the FRED is not approved currently. Still, in a comparative study of EuFRED anterior circulation aneurysms matched to aneurysms treated with the PED at US centers, both safety and efficacy profiles 
Table 2: Treatment characteristics and outcomes

\begin{tabular}{|c|c|}
\hline Parameter & No. \\
\hline \multicolumn{2}{|l|}{ No. of FREDs per aneurysm } \\
\hline 1 FRED & $80(95.2 \%)$ \\
\hline 2 FREDs & $3(3.6 \%)$ \\
\hline 4 FREDs & $1(1.2 \%)$ \\
\hline Adjunctive coiling required & $14(16.7 \%)$ \\
\hline Platelet function testing & $80(95.2 \%)$ \\
\hline Antiplatelet regimen changed after testing & $2(2.4 \%)$ \\
\hline \multicolumn{2}{|l|}{ Postprocedural antiplatelet regimen } \\
\hline ASA + clopidogrel & $62(73.8 \%)$ \\
\hline Tirofiban loading followed by ASA + clopidogrel & $11(13.1 \%)$ \\
\hline ASA + ticagrelor & $6(7.1 \%)$ \\
\hline Prasugrel & $3(3.6 \%)$ \\
\hline ASA + prasugrel & $1(1.2 \%)$ \\
\hline ASA + tirofiban + ticagrelor & $1(1.2 \%)$ \\
\hline Thromboembolic infarction due to procedure & $11(13.1 \%)$ \\
\hline Symptomatic thromboembolic complication & $8(9.5 \%)$ \\
\hline \multicolumn{2}{|l|}{ Location of infarction } \\
\hline Cerebellum & $7(8.3 \%)$ \\
\hline Brain stem & $2(2.4 \%)$ \\
\hline Posterior cerebral artery territory & $1(1.2 \%)$ \\
\hline Thalamus & $1(1.2 \%)$ \\
\hline Hemorrhagic complications & $0(0.0 \%)$ \\
\hline Other complications & $3(3.6 \%)$ \\
\hline Parent artery occlusion & $2(2.4 \%)$ \\
\hline $\begin{array}{l}\text { Time of radiographic follow-up (median) } \\
\text { (range) (mo) }\end{array}$ & $24(0.03-72)$ \\
\hline \multicolumn{2}{|l|}{ Occlusion status last imaging follow-up ${ }^{a}$} \\
\hline Complete occlusion (100\%) & $61(78.2 \%)$ \\
\hline Near-complete occlusion (>90\%) & $7(9 \%)$ \\
\hline Incomplete occlusion $(<90 \%)$ & $10(12.8 \%)$ \\
\hline \multicolumn{2}{|l|}{ Imaging technique ${ }^{\mathrm{b}}$} \\
\hline MRA & $34(50 \%)$ \\
\hline DSA & $31(45.6 \%)$ \\
\hline Conebeam CT with IV contrast & $2(2.9 \%)$ \\
\hline CTA & $1(1.5 \%)$ \\
\hline Retreatment $^{c}$ & $6(7.1 \%)$ \\
\hline Time of functional outcome follow-up (median) & $27(0.03-72)$ \\
\hline \multicolumn{2}{|l|}{ Functional outcome } \\
\hline mRS 0-2 & $78(94 \%)$ \\
\hline mRS 3-5 & 0 \\
\hline mRS 6 & $5(6 \%)$ \\
\hline
\end{tabular}

Note:-ASA indicates acetylsalicylic acid.

${ }^{a}$ Data missing in 6 patients.

${ }^{\mathrm{b}}$ Data missing in 16 patients.

c Data missing in 1 patient.

${ }^{\mathrm{d}}$ Data missing in 6 patients.

e Data missing in 1 patient.

of both flow diverters were comparable. ${ }^{10}$ To overcome the limitation of short angiographic follow-up of 6.6 months in the initial EuFRED publication, ${ }^{7}$ we updated both angiographic and clinical follow-up for the current study. With median follow-ups of 24 and 27 months, respectively, this study also presents some of the longest follow-up data on the FRED. ${ }^{8,9}$

\section{Occlusion of Posterior Circulation Aneurysms with Flow Diverters}

Per FDA regulations, flow diversion in the posterior circulation continues to be considered off-label. Nevertheless, flow diversion is increasingly used for posterior circulation aneurysms with most data available for the PED. ${ }^{4-6,11}$

The largest single-center series on posterior circulation aneurysms treated with either the PED or the p64 (phenox, Bochum,
Germany) included 58 nonsaccular aneurysms. ${ }^{11}$ The cohort represents the most challenging aneurysm morphologies in the posterior circulation. ${ }^{6}$ Angiographic occlusion or minor remnants were reported in $100 \%(5 / 5)$ of dolichoectatic, $95.5 \%(21 / 22)$ of fusiform, and $63.2 \%(12 / 19)$ of transitional morphology at a mean follow-up of 25.2 months. Up to 8.3 flow diverters were used, on average, in fusiform basilar artery aneurysms. A metaanalysis of nonsaccular posterior circulation aneurysms treated with flow diverters reported the average device number at 4.33 per aneurysm. ${ }^{6}$ In contrast, the present study included 50 nonsaccular aneurysms classified as fusiform, dissecting, and blister, with a median diameter of $7 \mathrm{~mm}$. The median number of FREDs used was 1. Among patients with angiographic follow-up and nonsaccular aneurysms, the rates of complete and near-complete occlusion were $81.8 \%$ and $4.6 \%$, respectively, at 18.5 months. Given the complexity of these lesions, however, any comparison must be interpreted with caution. Still, data are promising in terms of occlusion of some of the most challenging cerebral aneurysms.

The second largest single-center series was published in 2019, including 55 aneurysms treated with the PED. ${ }^{4}$ The classification of aneurysms was similar to that in the current study as saccular, fusiform, and dissecting/pseudoaneurysm. One PED was used in $84 \%$ of procedures. Angiographic follow-up was short, with only 8 aneurysms imaged at 24 months. On last follow-up, however, complete occlusion or trace filling was achieved in $72 \%$ and $9 \%$, respectively, which is comparable with the present study, especially given the shorter follow-up.

The largest series on posterior circulation flow diversion was a multicenter study, including 131 aneurysms, all treated with the PED. ${ }^{5}$ At a median of 11 months, complete or near-complete occlusion was achieved in $78.1 \%$ of aneurysms, with dissecting and fusiform aneurysms occluding most and least frequently, respectively. A predictor of occlusion in dissecting aneurysms was age, with older aneurysms occluding less often, a relationship that has also been demonstrated for anterior circulation aneurysms after flow diversion. ${ }^{12}$ In fusiform aneurysms, no predictors of occlusion were identified. Assessed variables were aneurysm size and location, intraluminal thrombus, adjunctive coiling, and the number of devices used. Saccular aneurysms of the basilar artery occluded less often than saccular aneurysms in other posterior circulation locations, though this relationship was not significant $(P=.08) .^{5}$

\section{Complications Associated with Posterior Circulation Flow Diversion}

The posterior circulation is characterized by unique neurovascular anatomy with end arterial perforating vessels to the brain stem arising from the distal vertebral artery, basilar trunk, and proximal posterior cerebral arteries. Placement of flow diverters inevitably results in coverage of smaller or larger side branches of the vertebrobasilar system. ${ }^{13}$

Reported morbidity and mortality are important. An aforementioned meta-analysis of 131 posterior circulation aneurysms treated with flow diversion reported overall morbidity and mortality rates of $26 \%$ and $21 \%$, respectively. Favorable clinical outcome (mRS, $\leq 2)$ was most frequently achieved in the vertebral 
artery as opposed to other locations, likely owing to the relative paucity of perforators in that location. ${ }^{6}$ In the present study, 11 thromboembolic complications occurred. Even though there was not a predilection for a specific location, 10 of 11 (90.9\%) thromboembolic complications occurred in nonsaccular aneurysms, and 5 of 11 (45.5\%), in aneurysms that had previously ruptured.

The multicenter study of 131 posterior circulation aneurysms treated with the PED also identified fusiform aneurysm morphology as a risk factor for major complications. ${ }^{5}$ Nevertheless, data from the present study show that even nonsaccular aneurysms of the posterior circulation can be treated safely with flow diverters as long as treatment is performed in unruptured aneurysms. Several key aspects, as summarized by Bhogal et al, ${ }^{11}$ are critical to minimize complications. These include rigorous platelet function testing and regimen adjustments, performed in $95.2 \%$ of patients in the current study; treatment of not acutely symptomatic patients, either due to rupture or brain stem compression or ischemia; the use of fewer flow diverters per aneurysm (median of 1 in the current series); and adjunctive coiling. Applying those principles in the current study may have explained the very favorable safety profile.

\section{Strengths and Limitations}

The most significant limitation is the heterogeneity of posterior circulation aneurysms. Given their variability in size and involvement of various segments of the posterior circulation tree, comparative analyses are challenging. Thus, any related study is exposed to significant selection bias across different neurovascular centers and individual neurovascular practitioners. Data collection was performed at the individual institution and was not core lab-adjudicated, introducing potential bias. Nevertheless, the dataset was nearly complete. The inclusion of multiple institutions, on the other hand, improved the generalizability of the findings. Another limitation was variability in the type of platelet function test used at the individual institution, albeit most patients underwent platelet function testing.

\section{CONCLUSIONS}

The largest cohort of posterior circulation aneurysms treated with the FRED to date demonstrated favorable safety and efficacy profiles of the device for this indication. Regardless of location and morphology, flow diversion using the FRED represents a viable treatment option for those challenging lesions. Thromboembolic complications occurred mostly in nonsaccular aneurysms. Treatment in the setting of acute subarachnoid hemorrhage, however, was strongly related to mortality, regardless of whether procedural complications occurred.

Disclosures: Markus A. Möhlenbruch—UNRELATED: Board Membership: Codman Neurovascular; Consultancy: Medtronic, MicroVention, Stryker*; Grants/Grants Pending: Balt, MicroVention*; Payment for Lectures Including Service on Speakers Bureaus: Medtronic, MicroVention, phenox, Stryker.* Christian UlfertUNRELATED: Payment for Lectures Including Service on Speakers Bureaus: Cerenovus; Travel/Accommodations/Meeting Expenses Unrelated to Activities Listed: Stryker, MicroVention, Balt. Markus Holtmannspötter-UNRELATED: Consultancy: Medtronic, MicroVention, Sequent Medical, Stryker, Mentice, Route 92, Comments: consultancy and proctoring agreement; Payment for Lectures Including Service on Speakers Bureaus: Medtronic, MicroVention, Sequent Medical. Jan-Hendrik
Buhk—UNRELATED: Consultancy: Acandis, Cerenovus, Codman Neurovascular, Medtronic, MicroVention, Stryker, Comments: consultancy and proctoring. Naci Kocer -UNRELATED: Consultancy: MicroVention, Medtronic, Comments: consultant and proctorship agreement; Payment for Lectures Including Service on Speakers Bureaus: MicroVention, Comments: lectures on FRED. Hendrik Janssen-UNRELATED: Consultancy: MicroVention/Sequent Medical, Comments: proctor*; Travel/ Accommodations/Meeting Expenses Unrelated to Activities Listed: MicroVention, Comments: travel expenses for conferences.* Monika KillerOberpfalzer-RELATED: Grant: MicroVention/Terumo*; Consulting Fee or Honorarium: MicroVention/Terumo; UNRELATED: Consultancy: Medtronic, Stryker, Cerus; Grant: MicroVention/Terumo, Comments: research grant*; Support for Travel to Meetings for the Study or Other Purposes: MicroVention/Terumo; Travel/Accommodations/Meeting Expenses Unrelated to Activities Listed: MicroVention, Stryker, Medtronic. Civan IslakUNRELATED: Consultancy: MicroVention; Patents (Planned, Pending or Issued): US 2015/0327868A1. *Money paid to institution.

\section{REFERENCES}

1. Petr $\mathrm{O}$, Brinjikji $\mathrm{W}$, Cloft $\mathrm{H}$, et al. Current trends and results of endovascular treatment of unruptured intracranial aneurysms at a single institution in the flow-diverter era. AJNR Am J Neuroradiol 2016;37:1106-13 CrossRef Medline

2. Ogilvy CS, Jordan NJ, Ascanio LC, et al. Surgical and endovascular comprehensive treatment outcomes of unruptured intracranial aneurysms: reduction of treatment bias. World Neurosurg 2019;126: e878-87 CrossRef Medline

3. Wang CB, Shi WW, Zhang GX, et al. Flow diverter treatment of posterior circulation aneurysms: a meta-analysis. Neuroradiology 2016;58:391-400 CrossRef Medline

4. Bender MT, Colby GP, Jiang B, et al. Flow diversion of posterior circulation cerebral aneurysms: a single-institution series of 59 cases. Neurosurgery 2019;84:206-16 CrossRef Medline

5. Griessenauer CJ, Ogilvy CS, Adeeb N, et al. Pipeline embolization of posterior circulation aneurysms: a multicenter study of 131 aneurysms. J Neurosurg 2018;130:923-35 CrossRef Medline

6. Kiyofuji S, Graffeo CS, Perry A, et al. Meta-analysis of treatment outcomes of posterior circulation non-saccular aneurysms by flow diverters. J Neurointerv Surg 2018;10:493-99 CrossRef Medline

7. Killer-Oberpfalzer M, Kocer N, Griessenauer CJ, et al. European multicenter study for the evaluation of a dual-layer flow-diverting stent for treatment of wide-neck intracranial aneurysms: the European Flow-Redirection Intraluminal Device Study. AJNR Am J Neuroradiol 2018;39:841-47 CrossRef Medline

8. Piano M, Valvassori L, Lozupone E, et al. FRED Italian Registry: a multicenter experience with the Flow Re-direction Endoluminal Device for intracranial aneurysms. J Neurosurg 2019;1-8 CrossRef Medline

9. Pierot L, Spelle L, Berge J, et al. SAFE study (Safety and efficacy Analysis of FRED Embolic device in aneurysm treatment): 1-year clinical and anatomical results. J Neurointerv Surg 2019;11:184-89 CrossRef Medline

10. Griessenauer CJ, Thomas AJ, Enriquez-Marulanda A, et al. Comparison of Pipeline Embolization Device and Flow Re-Direction Endoluminal Device flow diverters for internal carotid artery aneurysms: a propensity score-matched cohort study. Neurosurgery 2019;85: E249-55 CrossRef Medline

11. Bhogal P, Pérez MA, Ganslandt O, et al. Treatment of posterior circulation non-saccular aneurysms with flow diverters: a single-center experience and review of 56 patients. J Neurointerv Surg 2017; 9:471-81 CrossRef Medline

12. Adeeb N, Moore JM, Wirtz M, et al. Predictors of incomplete occlusion following Pipeline embolization of intracranial aneurysms: is it less effective in older patients? AJNR Am J Neuroradiol 2017;38:2295-300 CrossRef Medline

13. Adeeb N, Griessenauer CJ, Dmytriw AA, et al. Risk of branch occlusion and ischemic complications with the Pipeline embolization device in the treatment of posterior circulation aneurysms. AJNR Am J Neuroradiol 2018;39:1303-09 CrossRef Medline 